\title{
ANTIPLATELET THERAPY
}

\section{Treatment with ticagrelor for 12 months after ACS is cost-effective}

Antiplatelet therapy with ticagrelor for 12 months in patients with either ST-segment elevation or non-ST-segment elevation acute coronary syndrome (ACS) is cost-effective in the long term compared with generic clopidogrel. This finding comes from the Health Economic Substudy Group of the Platelet Inhibition and Patients Outcomes (PLATO) trial, and is published in the European Heart Journal.

In total, 18,624 patients with ACS were randomly allocated to either ticagrelor plus aspirin or clopidogrel plus aspirin in the PLATO trial. Ticagrelor treatment reduced the composite end point of death from vascular causes, myocardial infarction, or stroke (HR 0.84, 95\% CI $0.77-0.92, P<0.001)$ without increasing the rate of major bleeding. In the two-part cost-effectiveness analysis, data from the PLATO trial were used in a short-term (1-year) decision tree, and then a Markov model was used to extrapolate long-term costs and efficacy in the Swedish health-care setting. Ticagrelor and generic clopidogrel were estimated to cost $€ 2.21$ and $€ 0.06$ per day, respectively.

Ticagrelor therapy was associated with a quality-adjusted life-year gain of 0.1316 at an incremental cost of $€ 362$. Therefore, costs were estimated to be around $€ 2,372$ and $€ 2,753$ per life-year or quality-adjusted life-year gained, respectively, compared with clopidogrel treatment. These values were driven predominantly by the reduction in mortality with ticagrelor reported in the PLATO trial, and are below the generally accepted thresholds for cost-effectiveness.

Gregory B. Lim

Original article Nikolic, E. et al. Cost-effectiveness of treating acute coronary syndrome patients with ticagrelor for 12 months: results from the PLATO study. Eur. Heart J. doi:10.1093/eurheartj/ehs149 